\title{
Risk of antimicrobial-associated organ injury among the older adults: a systematic review and meta-analysis
}

\author{
Tichawona Chinzowu ${ }^{1 *}$, Sandipan Roy ${ }^{2}$ and Prasad S. Nishtala
}

\begin{abstract}
Background: Older adults (aged 65 years and above) constitute the fastest growing population cohort in the western world. There is increasing evidence that the burden of infections disproportionately affects older adults, and hence this vulnerable population is frequently exposed to antimicrobials. There is currently no systematic review summarising the evidence for organ injury risk among older adults following antimicrobial exposure. This systematic review and metaanalysis examined the relationship between antimicrobial exposure and organ injury in older adults.
\end{abstract}

Methodology: We searched for original research articles in PubMed, Embase.com, Web of Science core collection, Web of Science BIOSIS citation index, Scopus, Cochrane Central Register of Controlled Trials, ProQuest, and PsycINFO databases, using key words in titles and abstracts, and using MeSH terms. We searched for all available articles up to 31 May 2021. After removing duplicates, articles were screened for inclusion into or exclusion from the study by two reviewers. The Newcastle-Ottawa scale was used to assess the risk of bias for cohort and case-control studies. We explored the heterogeneity of the included studies using the $\mathrm{Q}$ test and $\mathrm{I}^{2}$ test and the publication bias using the funnel plot and Egger's test. The meta-analyses were performed using the OpenMetaAnalyst software.

Results: The overall absolute risks of acute kidney injury among older adults prescribed aminoglycosides, glycopeptides, and macrolides were 15.1\% (95\% Cl: 12.8-17.3), 19.1\% (95\% Cl: 15.4-22.7), and 0.3\% (95\% Cl: 0.3-0.3), respectively. Only 3 studies reported antimicrobial associated drug-induced liver injury. Studies reporting on the association of organ injury and antimicrobial exposure by age or duration of treatment were too few to meta-analyse. The funnel plot and Egger's tests did not indicate evidence of publication bias.

Conclusion: Older adults have a significantly higher risk of sustaining acute kidney injury when compared to the general adult population. Older adults prescribed aminoglycosides have a similar risk of acute kidney injury to the general adult population.

Keywords: Systematic review, meta-analysis, Older adults, Antimicrobial, Organ injury

\footnotetext{
* Correspondence: tc888@bath.ac.uk

'Department of Pharmacy and Pharmacology \& Centre for Therapeutic Innovation, University of Bath, Bath BA2 7AY, UK

Full list of author information is available at the end of the article
}

(C) The Author(s). 2021 Open Access This article is licensed under a Creative Commons Attribution 4.0 International License, which permits use, sharing, adaptation, distribution and reproduction in any medium or format, as long as you give appropriate credit to the original author(s) and the source, provide a link to the Creative Commons licence, and indicate if changes were made. The images or other third party material in this article are included in the article's Creative Commons licence, unless indicated otherwise in a credit line to the material. If material is not included in the article's Creative Commons licence and your intended use is not permitted by statutory regulation or exceeds the permitted use, you will need to obtain permission directly from the copyright holder. To view a copy of this licence, visit http://creativecommons.org/licenses/by/4.0/ The Creative Commons Public Domain Dedication waiver (http://creativecommons.org/publicdomain/zero/1.0/) applies to the data made available in this article, unless otherwise stated in a credit line to the data. 


\section{Background}

Older adults aged 65 years and above comprise the fastest and largest expanding population age group in the developed world [1]. They are prone to infectious diseases such as pneumonia, skin and soft tissue infections (SSTI), urinary tract infections (UTI) and septicaemia when compared to younger people [1]. It is estimated that the older adults comprise $48.7 \%$ of individuals admitted to hospital intensive care units for these infections [2], resulting in their increased length of hospital stay and exposure to antimicrobials. Giarratano et al. [3] highlighted several predisposing factors that make older adults more susceptible to antimicrobial adverse events. These include physiological changes, higher comorbidities, drug-drug interactions, drug delivery routes used, and length of time they are in contact or exposed to the antimicrobial agents. In one large prospective cohort study, antimicrobial related adverse events accounted for $19.3 \%$ of all drug-related adverse events seen at the emergency department [2]. Several antimicrobialassociated adverse events become apparent years after the drug has been approved. The adverse events reported in clinical trials differ considerably from post-marketing surveillance [4]. Since most clinical trials exclude older adults, the true nature and incidence of antimicrobial related adverse events in this population are unknown. In their review, Giarratano et al. [3] concluded that there is a general lack of epidemiological studies on antimicrobials used among the older adults, yet this is essential in informing healthcare providers to achieve optimal safety and effectiveness when providing antimicrobial pharmacotherapy to the older adults.

This paper describes a systematic review and metaanalysis carried out to investigate the risk of antimicrobial-associated kidney, liver, or tissue injury among older adults. The main outcome was quantifying the association of organ injury among older adults following exposure to antimicrobials.

\section{Methods}

This systematic review was conducted after the study protocol was registered with PROSPERO (CRD4202015262).

\section{Search strategy and selection criteria}

For this systematic review and meta-analysis, we searched for original research articles for observational studies describing kidney, liver or tissue injury associated with exposure to antimicrobials among older adults (65 years or above). We restricted our searches to the English language only for all available articles up to 31 May 2021.

Searches were conducted in PubMed, Embase.com, Web of Science core collection, Web of Science BIOSIS citation index, Scopus, Cochrane Central Register of Controlled Trials, ProQuest, and PsycINFO databases, using key words in titles and abstracts, and using $\mathrm{MeSH}$ terms. The full search criteria for all the databases are in additional file 1 , document 1 . After removing duplicates, TC carried out the title and abstract screening on all the articles for preliminary inclusion into the systematic review. PN and SR independently carried out title and abstract screening of $50 \%$ of the articles each for preliminary inclusion into the systematic review. Among the preliminary included articles, TC and PN carried out full article review of the first $50 \%$ of the articles, and TC and SR reviewed the other half. Where there were disagreements between reviewers in both the preliminary review and full article review, the opinion of the third reviewer was sought.

\section{Inclusion and exclusion criteria}

Studies were included in the systematic review and metaanalysis if they reported the absolute risk of antimicrobialassociated kidney, liver, or tissue injury among older adults. Studies were also included if they reported a subgroup analysis of participants who met the inclusion criteria, thus 65 years or older. Studies were excluded if they included adults below 65 years of age without relevant subgroup analysis for those 65 years or older; the outcome of interest was not kidney, liver, or tissue injury; the exposure was not an antimicrobial; and the study design was not an original observational study.

\section{Risk of bias assessment}

The Newcastle-Ottawa scale [5] was used to assess the risk of bias for each observational study (see additional file 4, document 1). Each study that scored 6 or more points on the Newcastle-Ottawa scale was considered to have a low risk of bias provided it scored at least 3 points on the selection domain, one or more on comparability, and at least 2 points on the outcome domain. All studies considered to be of low risk to bias were further considered for meta-analysis.

\section{Data analysis}

Data extraction was completed by TC and PN independently, using a pre-piloted excel form. Data for the following variables were extracted: author details, country of study, study design, study setting, data source, any condition treated with antimicrobial, inclusion and exclusion criteria, outcome measurement and ascertainment, controlled confounders, sample size, exposure antibiotic, and absolute risk or odds ratio of organ injury among the participants. The full data extraction table is available as additional file 2, document 1 .

Heterogeneity among the studies included for metaanalysis was assessed using the Cochrane Q statistic [6] at $5 \%$ significance level and quantified using Higgins and Thompson's $\mathrm{I}^{2}$ statistic [6]. An $\mathrm{I}^{2}$ value of more than $50 \%$ was considered to reflect substantial heterogeneity, 
and therefore, sensitivity analysis to investigate the possible source of heterogeneity was done. For the metaanalysis results, publication bias was assessed using a funnel plot [7] and Egger's regression test [8]. Regardless of the heterogeneity, the random-effects model, using the DerSimonian and Laird method [9], was used to meta-analyse the studies. The meta-analyses were performed using the OpenMetaAnalyst software [10].

\section{Results}

\section{Study identification}

Following database searching, 10,320 studies were identified from the following databases: PubMed (1164), Embase.com (4880), Web of Science core collection (250), Web of
Science BIOSIS citation index (301), Scopus (2901), Cochrane Central Register of Controlled Trials (817), ProQuest (6), and PsycINFO (1), of which 2758 were duplicates. Of the remaining 7562 studies, 7237 studies were excluded following title and abstract screening. A further 296 studies were excluded after the full-text screening, leaving 29 studies for inclusion into the the systematic review [11-39]. The full selection process is summarised in Fig. 1 below. Six of the included studies had data for two or more antimicrobials [16-18, 24, 27, 37], as shown in Table 1 below.

\section{Characteristics of included studies}

Out of the 29 studies included for qualitative analysis, eight were prospective cohort studies [12, 22, 29-31, 33,

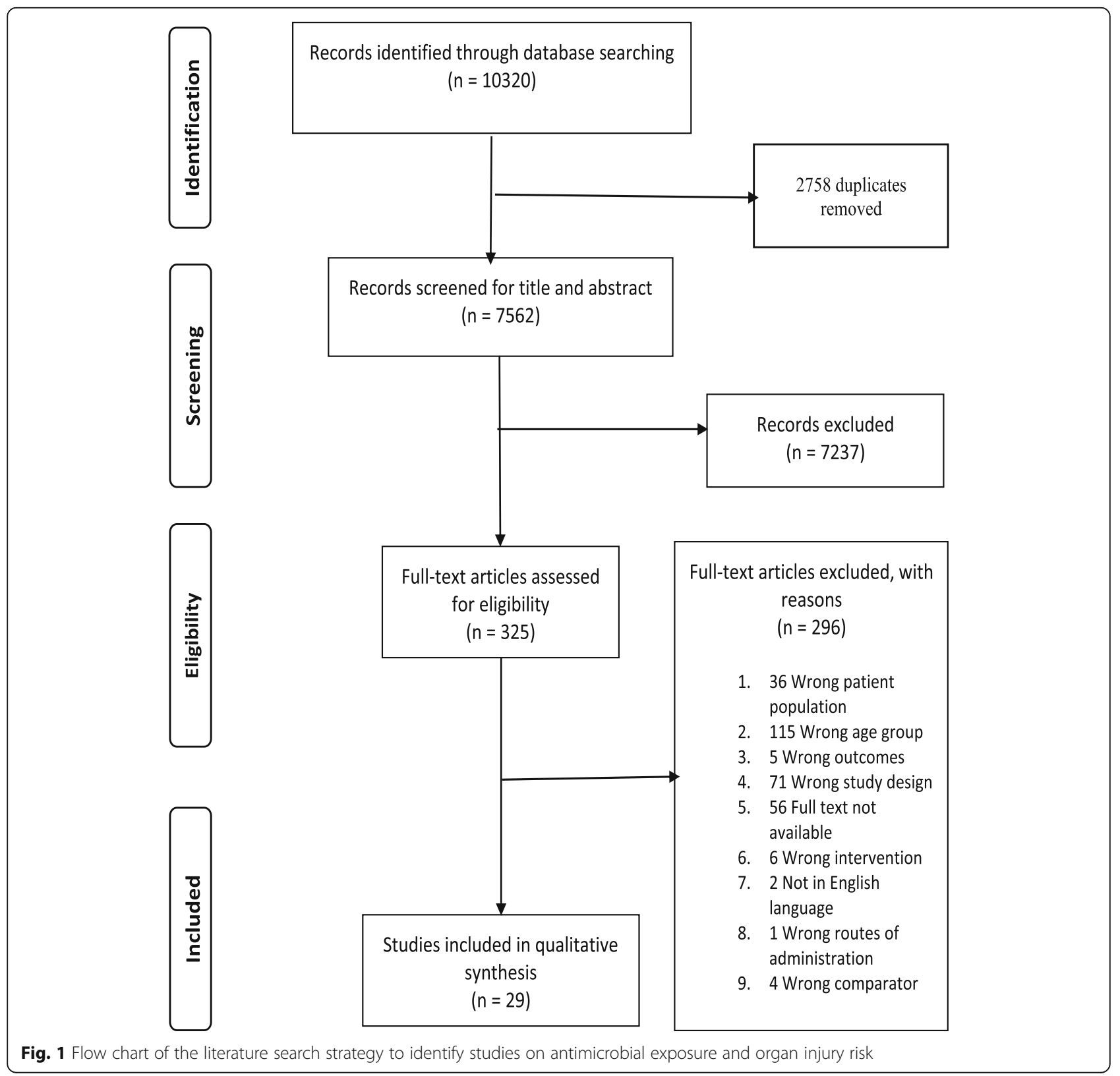


Table 1 Main characteristics of the included studies on antimicrobials and organ injury

\begin{tabular}{|c|c|c|c|c|c|c|c|c|c|}
\hline $\begin{array}{l}\text { Author, } \\
\text { Year }\end{array}$ & Country & Method & $\begin{array}{l}\text { Study } \\
\text { Setting }\end{array}$ & $\begin{array}{l}\text { Tool used to } \\
\text { ascertain the } \\
\text { outcome }\end{array}$ & Confounders & $\begin{array}{l}\text { Sample } \\
\text { size }\end{array}$ & Antibiotic class & Outcome & $\begin{array}{l}\text { NOS } \\
\text { Risk of } \\
\text { bias }\end{array}$ \\
\hline $\begin{array}{l}\text { Aggarwal, } \\
2018 \text { [31] }\end{array}$ & India & $\begin{array}{l}\text { Prospective } \\
\text { cohort }\end{array}$ & $\begin{array}{l}\text { Hospital } \\
\text { setting }\end{array}$ & $\begin{array}{l}\text { Laboratory } \\
\text { values }\end{array}$ & $\begin{array}{l}\text { Age, gender, BMl, } \\
\text { APACHE 2, DM, antibiotic } \\
\text { dose }\end{array}$ & 26 & Polymyxin & AKI & Low \\
\hline $\begin{array}{l}\text { Ahmed, } \\
2016 \text { [38] }\end{array}$ & UK & $\begin{array}{l}\text { Retrospective } \\
\text { cohort }\end{array}$ & $\begin{array}{l}\text { Hospital } \\
\text { setting }\end{array}$ & AKIN & $\begin{array}{l}\text { age, gender, surgeon } \\
\text { grade, type of } \\
\text { anaesthesia }\end{array}$ & 1500 & Aminoglycoside & AKI & Low \\
\hline $\begin{array}{l}\text { Ahmed, } \\
2019 \text { [37] }\end{array}$ & UK & $\begin{array}{l}\text { Retrospective } \\
\text { cohort }\end{array}$ & $\begin{array}{l}\text { Primary } \\
\text { care }\end{array}$ & ICD-10 codes & $\begin{array}{l}\text { age, sex, coronary heart } \\
\text { disease, renal disease, } \\
\text { liver disease, respiratory } \\
\text { disease, type } 2 \text { diabetes } \\
\text { mellitus, heart failure }\end{array}$ & 28,800 & Cephalosporin & AKI & Low \\
\hline $\begin{array}{l}\text { Ahmed, } \\
2019 \text { [37] }\end{array}$ & UK & $\begin{array}{l}\text { Retrospective } \\
\text { cohort }\end{array}$ & $\begin{array}{l}\text { Primary } \\
\text { care }\end{array}$ & ICD-10 codes & $\begin{array}{l}\text { age, sex, coronary heart } \\
\text { disease, renal disease, } \\
\text { liver disease, respiratory } \\
\text { disease, type } 2 \text { diabetes } \\
\text { mellitus, heart failure }\end{array}$ & 21,048 & Penicillin & AKI & Low \\
\hline $\begin{array}{l}\text { Ahmed, } \\
2019 \text { [37] }\end{array}$ & UK & $\begin{array}{l}\text { Retrospective } \\
\text { cohort }\end{array}$ & $\begin{array}{l}\text { Primary } \\
\text { care }\end{array}$ & ICD-10 codes & $\begin{array}{l}\text { age, sex, coronary heart } \\
\text { disease, renal disease, } \\
\text { liver disease, respiratory } \\
\text { disease, type } 2 \text { diabetes } \\
\text { mellitus, heart failure }\end{array}$ & 14,868 & Quinolone & AKI & Low \\
\hline $\begin{array}{l}\text { Ahmed, } \\
2018 \text { [11] }\end{array}$ & UK & $\begin{array}{l}\text { Retrospective } \\
\text { cohort }\end{array}$ & $\begin{array}{l}\text { Primary } \\
\text { care }\end{array}$ & ICD-10 codes & $\begin{array}{l}\text { age, diabetes, dementia, } \\
\text { coronary heart disease, } \\
\text { stroke, cancer, heart } \\
\text { failure, polypharmacy }\end{array}$ & 29,936 & Trimethoprim & AKI & Low \\
\hline $\begin{array}{l}\text { Baciewicz, } \\
2003[12]\end{array}$ & USA & $\begin{array}{l}\text { Prospective } \\
\text { cohort }\end{array}$ & $\begin{array}{l}\text { Hospital } \\
\text { setting }\end{array}$ & $\begin{array}{l}\text { Laboratory } \\
\text { values }\end{array}$ & $\begin{array}{l}\text { age, sex, nephrotoxic } \\
\text { agents, diabetes mellitus, } \\
\text { coronary artery disease, } \\
\text { hypertension and }\end{array}$ & 86 & Aminoglycoside & AKI & Low \\
\hline $\begin{array}{l}\text { Baghaei, } \\
2010 \text { [33] }\end{array}$ & Iran & $\begin{array}{l}\text { Prospective } \\
\text { cohort }\end{array}$ & $\begin{array}{l}\text { Hospital } \\
\text { setting }\end{array}$ & $\begin{array}{l}\text { Laboratory } \\
\text { values }\end{array}$ & $\begin{array}{l}\text { Age, sex, concomitant } \\
\text { hepatotoxic drugs }\end{array}$ & 295 & antituberculosis & DILI & Low \\
\hline $\begin{array}{l}\text { Baik, } 2020 \\
{[36]}\end{array}$ & USA & $\begin{array}{l}\text { Prospective } \\
\text { cohort }\end{array}$ & $\begin{array}{l}\text { Population } \\
\text { based }\end{array}$ & ICD-9 codes & $\begin{array}{l}\text { Concurrent antibiotics, } \\
\text { gender, race, rural } \\
\text { residency, income status }\end{array}$ & 685,018 & Fluoroquinolone & $\begin{array}{l}\text { Tendon } \\
\text { rupture }\end{array}$ & Low \\
\hline $\begin{array}{l}\text { Bright- } \\
\text { Thomas, } \\
2016 \text { [13] }\end{array}$ & UK & $\begin{array}{l}\text { Retrospective } \\
\text { cohort }\end{array}$ & $\begin{array}{l}\text { Primary } \\
\text { care }\end{array}$ & $\begin{array}{l}\text { Laboratory } \\
\text { values }\end{array}$ & age, sex, ethnic origin & 200 & $\begin{array}{l}\text { Antituberculosis } \\
\text { drugs }\end{array}$ & DILI & Low \\
\hline $\begin{array}{l}\text { Carreno, } \\
2013[14]\end{array}$ & USA & $\begin{array}{l}\text { Retrospective } \\
\text { cohort }\end{array}$ & $\begin{array}{l}\text { Hospital } \\
\text { setting }\end{array}$ & AKIN & $\begin{array}{l}\text { age, history of AKI, } \\
\text { vancomycin dose, } \\
\text { concurrent receipt of } \\
\text { nephrotoxins, concurrent } \\
\text { receipt of vasopressors }\end{array}$ & 88 & Glycopeptide & AKI & Low \\
\hline $\begin{array}{l}\text { Craig, } 2012 \\
{[15]}\end{array}$ & UK & $\begin{array}{l}\text { Retrospective } \\
\text { cohort }\end{array}$ & $\begin{array}{l}\text { Hospital } \\
\text { setting }\end{array}$ & Not stated & $\begin{array}{l}\text { age, sex, operative } \\
\text { procedure }\end{array}$ & 200 & Aminoglycoside & AKI & Low \\
\hline $\begin{array}{l}\text { Crellin, } \\
2018[16]\end{array}$ & UK & $\begin{array}{l}\text { Retrospective } \\
\text { cohort }\end{array}$ & $\begin{array}{l}\text { Multicentre } \\
\text { Primary } \\
\text { care }\end{array}$ & ICD-10 codes & $\begin{array}{l}\text { sex, age, calendar period, } \\
\text { chronic comorbidities, } \\
\text { baseline renal function, } \\
\text { history of renal or } \\
\text { urological disease, and } \\
\text { use of renin-angiotensin } \\
\text { system blockers and } \\
\text { potassium-sparing } \\
\text { diuretics. }\end{array}$ & 55,961 & Cephalosporin & AKI & Low \\
\hline $\begin{array}{l}\text { Crellin, } \\
2018[16]\end{array}$ & UK & $\begin{array}{l}\text { Retrospective } \\
\text { cohort }\end{array}$ & $\begin{array}{l}\text { Multicentre } \\
\text { Primary } \\
\text { care }\end{array}$ & ICD-10 codes & $\begin{array}{l}\text { sex, age, calendar period, } \\
\text { chronic comorbidities, } \\
\text { baseline renal function, } \\
\text { history of renal or } \\
\text { urological disease, and } \\
\text { use of renin-angiotensin }\end{array}$ & 56,736 & Nitrofurantoin & AKI & Low \\
\hline
\end{tabular}


Table 1 Main characteristics of the included studies on antimicrobials and organ injury (Continued)

\begin{tabular}{|c|c|c|c|c|c|c|c|c|c|}
\hline $\begin{array}{l}\text { Author, } \\
\text { Year }\end{array}$ & Country & Method & $\begin{array}{l}\text { Study } \\
\text { Setting }\end{array}$ & $\begin{array}{l}\text { Tool used to } \\
\text { ascertain the } \\
\text { outcome }\end{array}$ & Confounders & $\begin{array}{l}\text { Sample } \\
\text { size }\end{array}$ & Antibiotic class & Outcome & $\begin{array}{l}\text { NOS } \\
\text { Risk of } \\
\text { bias }\end{array}$ \\
\hline & & & & & $\begin{array}{l}\text { system blockers and } \\
\text { potassium-sparing } \\
\text { diuretics. }\end{array}$ & & & & \\
\hline $\begin{array}{l}\text { Crellin, } \\
2018[16]\end{array}$ & UK & $\begin{array}{l}\text { Retrospective } \\
\text { cohort }\end{array}$ & $\begin{array}{l}\text { Multicentre } \\
\text { Primary } \\
\text { care }\end{array}$ & ICD-10 codes & $\begin{array}{l}\text { sex, age, calendar period, } \\
\text { chronic comorbidities, } \\
\text { baseline renal function, } \\
\text { history of renal or } \\
\text { urological disease, and } \\
\text { use of renin-angiotensin } \\
\text { system blockers and } \\
\text { potassium-sparing } \\
\text { diuretics. }\end{array}$ & 33,130 & Quinolone & AKI & Low \\
\hline $\begin{array}{l}\text { Crellin, } \\
2018[16]\end{array}$ & UK & $\begin{array}{l}\text { Retrospective } \\
\text { cohort }\end{array}$ & $\begin{array}{l}\text { Multicentre } \\
\text { Primary } \\
\text { care }\end{array}$ & ICD-10 codes & $\begin{array}{l}\text { sex, age, calendar period, } \\
\text { chronic comorbidities, } \\
\text { baseline renal function, } \\
\text { history of renal or } \\
\text { urological disease, and } \\
\text { use of renin-angiotensin } \\
\text { system blockers and } \\
\text { potassium-sparing } \\
\text { diuretics. }\end{array}$ & 153,201 & Trimethoprim & AKI & Low \\
\hline $\begin{array}{l}\text { Fraisse, } \\
2014[17]\end{array}$ & France & $\begin{array}{l}\text { Retrospective } \\
\text { cohort }\end{array}$ & $\begin{array}{l}\text { Hospital } \\
\text { setting } \\
\text { multicentre }\end{array}$ & NKF & $\begin{array}{l}\text { age, sex, weight, } \\
\text { concomitant use of } \\
\text { other nephrotic drugs, } \\
\text { types of infection }\end{array}$ & 109 & Aminoglycoside & AKI & Low \\
\hline $\begin{array}{l}\text { Fraisse, } \\
2014[17]\end{array}$ & France & $\begin{array}{l}\text { Retrospective } \\
\text { cohort }\end{array}$ & $\begin{array}{l}\text { Hospital } \\
\text { setting } \\
\text { multicentre }\end{array}$ & NKF & $\begin{array}{l}\text { age, sex, weight, } \\
\text { concomitant use of } \\
\text { other nephrotic drugs, } \\
\text { types of infection }\end{array}$ & 45 & Aminoglycoside & AKI & Low \\
\hline $\begin{array}{l}\text { Gandhi, } \\
2013[18]\end{array}$ & Canada & $\begin{array}{l}\text { Retrospective } \\
\text { cohort }\end{array}$ & $\begin{array}{l}\text { Population- } \\
\text { based }\end{array}$ & ICD-10 codes & $\begin{array}{l}\text { age, sex, chronic kidney } \\
\text { disease, others }\end{array}$ & 96,226 & Macrolide & AKI & Low \\
\hline $\begin{array}{l}\text { Gandhi, } \\
2013[18]\end{array}$ & Canada & $\begin{array}{l}\text { Retrospective } \\
\text { cohort }\end{array}$ & $\begin{array}{l}\text { Population- } \\
\text { based }\end{array}$ & ICD-10 codes & $\begin{array}{l}\text { age, sex, chronic kidney } \\
\text { disease, others }\end{array}$ & 94,083 & Macrolide & AKI & Low \\
\hline $\begin{array}{l}\text { Giri, } 2016 \\
{[34]}\end{array}$ & India & $\begin{array}{l}\text { Prospective } \\
\text { cohort }\end{array}$ & $\begin{array}{l}\text { Prospective } \\
\text { cohort }\end{array}$ & $\begin{array}{l}\text { Laboratory } \\
\text { values }\end{array}$ & $\begin{array}{l}\text { Overweight, age, sex, } \\
\text { impaired renal status, } \\
\text { pregnancy, } \\
\text { immunocompromised }\end{array}$ & 26 & Aminoglycoside & AKI & High \\
\hline $\begin{array}{l}\text { Gyamlani, } \\
2019 \text { [19] }\end{array}$ & USA & $\begin{array}{l}\text { Retrospective } \\
\text { cohort }\end{array}$ & $\begin{array}{l}\text { Hospital } \\
\text { setting }\end{array}$ & $\begin{array}{l}\text { CKD-EPI } \\
\text { equation }\end{array}$ & $\begin{array}{l}\text { age, sex, comorbidities, } \\
\text { baseline eGFR, exposure } \\
\text { to nephrotoxic } \\
\text { medication }\end{array}$ & 22,057 & Glycopeptide & AKI & Low \\
\hline $\begin{array}{l}\text { Hall, } 2014 \\
{[20]}\end{array}$ & USA & $\begin{array}{l}\text { Retrospective } \\
\text { cohort }\end{array}$ & $\begin{array}{l}\text { Hospital } \\
\text { setting }\end{array}$ & $\begin{array}{l}\text { Laboratory } \\
\text { values }\end{array}$ & $\begin{array}{l}\text { age, sex, hospital stay, } \\
\text { concomitant use of } \\
\text { nephrotoxins }\end{array}$ & 92 & Glycopeptide & AKI & Low \\
\hline $\begin{array}{l}\text { Huang, } \\
2018 \text { [21] }\end{array}$ & China & $\begin{array}{l}\text { Retrospective } \\
\text { cohort }\end{array}$ & $\begin{array}{l}\text { Hospital } \\
\text { setting }\end{array}$ & AKIN & $\begin{array}{l}\text { age, sex, type of } \\
\text { infection, comorbidities, } \\
\text { APACH II score }\end{array}$ & 50 & Glycopeptide & AKI & Low \\
\hline $\begin{array}{l}\text { Karino, } \\
2014 \text { [22] }\end{array}$ & Japan & $\begin{array}{l}\text { Prospective } \\
\text { cohort }\end{array}$ & $\begin{array}{l}\text { Hospital } \\
\text { setting }\end{array}$ & $\begin{array}{l}\text { Categorical } \\
\text { scale of mild, } \\
\text { moderate, or } \\
\text { serious (state } \\
\text { recommended). }\end{array}$ & $\begin{array}{l}\text { sex, age, underlying } \\
\text { disease }\end{array}$ & 22 & Penicillin & AKI & Low \\
\hline $\begin{array}{l}\text { Li, } 2015 \\
{[24]}\end{array}$ & Canada & $\begin{array}{l}\text { Retrospective } \\
\text { cohort }\end{array}$ & $\begin{array}{l}\text { Population- } \\
\text { based }\end{array}$ & ICD-10 codes & $\begin{array}{l}\text { age, sex, baseline } \\
\text { evidence of CKD, CVD, } \\
\text { cancer, diabetes }\end{array}$ & 52,518 & Macrolide & AKI & Low \\
\hline $\begin{array}{l}\text { Li, } 2015 \\
{[24]}\end{array}$ & Canada & $\begin{array}{l}\text { Retrospective } \\
\text { cohort }\end{array}$ & $\begin{array}{l}\text { Population- } \\
\text { based }\end{array}$ & ICD-10 codes & $\begin{array}{l}\text { age, sex, baseline } \\
\text { evidence of CKD, CVD, }\end{array}$ & 51,523 & Macrolide & AKI & Low \\
\hline
\end{tabular}


Table 1 Main characteristics of the included studies on antimicrobials and organ injury (Continued)

\begin{tabular}{|c|c|c|c|c|c|c|c|c|c|}
\hline $\begin{array}{l}\text { Author, } \\
\text { Year }\end{array}$ & Country & Method & $\begin{array}{l}\text { Study } \\
\text { Setting }\end{array}$ & $\begin{array}{l}\text { Tool used to } \\
\text { ascertain the } \\
\text { outcome }\end{array}$ & Confounders & $\begin{array}{l}\text { Sample } \\
\text { size }\end{array}$ & Antibiotic class & Outcome & $\begin{array}{l}\text { NOS } \\
\text { Risk of } \\
\text { bias }\end{array}$ \\
\hline $\begin{array}{l}\text { Liu, } 2015 \\
{[23]}\end{array}$ & China & $\begin{array}{l}\text { Retrospective } \\
\text { cohort }\end{array}$ & $\begin{array}{l}\text { Hospital } \\
\text { setting }\end{array}$ & AKIN & $\begin{array}{l}\text { age, sex, weight, } \\
\text { comorbidities, } \\
\text { concomitant use of ACEl, } \\
\text { ARBs, NSAIDs, } \\
\text { aminoglycosides, } \\
\text { immunosuppressants }\end{array}$ & 124 & Glycopeptide & AKI & Low \\
\hline $\begin{array}{l}\text { Mizokami, } \\
2013[25]\end{array}$ & Japan & $\begin{array}{l}\text { Retrospective } \\
\text { cohort }\end{array}$ & $\begin{array}{l}\text { Hospital } \\
\text { setting }\end{array}$ & $\begin{array}{l}\text { Laboratory } \\
\text { values }\end{array}$ & $\begin{array}{l}\text { age, gender, infection } \\
\text { severity, body weight, } \\
\text { comorbidity index }\end{array}$ & 94 & Glycopeptide & AKI & Low \\
\hline $\begin{array}{l}\text { Morimoto, } \\
2017 \text { [32] }\end{array}$ & Japan & $\begin{array}{l}\text { Prospective } \\
\text { cohort }\end{array}$ & $\begin{array}{l}\text { Hospital } \\
\text { setting }\end{array}$ & KDIGO & $\begin{array}{l}\text { Age, gender, } \\
\text { concomitant antibiotics, } \\
\text { BMI, BUN, severity of } \\
\text { pneumonia }\end{array}$ & 57 & Penicillin & AKI & Moderate \\
\hline $\begin{array}{l}\text { Noh, } 2019 \\
{[26]}\end{array}$ & $\begin{array}{l}\text { South } \\
\text { Korea }\end{array}$ & $\begin{array}{l}\text { Retrospective } \\
\text { cohort }\end{array}$ & $\begin{array}{l}\text { Hospital } \\
\text { setting }\end{array}$ & $\begin{array}{l}\text { Laboratory } \\
\text { values }\end{array}$ & $\begin{array}{l}\text { age, sex, comorbidities } \\
\text { (e.g., hypertension, etc.) }\end{array}$ & 77 & $\begin{array}{l}\text { Antituberculosis } \\
\text { drugs }\end{array}$ & DILI & Low \\
\hline $\begin{array}{l}\text { Ong, } 2016 \\
{[27]}\end{array}$ & Singapore & $\begin{array}{l}\text { Retrospective } \\
\text { cohort }\end{array}$ & $\begin{array}{l}\text { Hospital- } \\
\text { based }\end{array}$ & KDIGO & $\begin{array}{l}\text { age, sex, critical illness, } \\
\text { comorbidities (e.g. } \\
\text { diabetes, etc.), } \\
\text { nephrotoxic medication }\end{array}$ & 194 & Aminoglycoside & AKI & Low \\
\hline $\begin{array}{l}\text { Ong, } 2016 \\
{[27]}\end{array}$ & Singapore & $\begin{array}{l}\text { Retrospective } \\
\text { cohort }\end{array}$ & $\begin{array}{l}\text { Hospital- } \\
\text { based }\end{array}$ & KDIGO & $\begin{array}{l}\text { age, sex, critical illness, } \\
\text { comorbidities (e.g. } \\
\text { diabetes, etc.), } \\
\text { nephrotoxic medication }\end{array}$ & 84 & Aminoglycoside & AKI & Low \\
\hline $\begin{array}{l}\text { Pan, } 2018 \\
\text { [39] }\end{array}$ & China & $\begin{array}{l}\text { Retrospective } \\
\text { cross- } \\
\text { sectional }\end{array}$ & $\begin{array}{l}\text { Hospital- } \\
\text { based }\end{array}$ & KDIGO & $\begin{array}{l}\text { age, gender, baseline } \\
\text { serum creatinine, } \\
\text { vasopressors, beta- } \\
\text { blockers, furosemide, car- } \\
\text { bapenems, ICU admit- } \\
\text { tance, etc. }\end{array}$ & 647 & Glycopeptide & AKI & Low \\
\hline $\begin{array}{l}\text { Pan, } 2017 \\
{[28]}\end{array}$ & China & $\begin{array}{l}\text { Retrospective } \\
\text { cohort }\end{array}$ & $\begin{array}{l}\text { Hospital- } \\
\text { based }\end{array}$ & KDIGO & $\begin{array}{l}\text { sex, age, baseline serum } \\
\text { creatinine, the reason for } \\
\text { vancomycin therapy, ICU } \\
\text { admittance, etc. }\end{array}$ & 279 & Glycopeptide & AKI & Low \\
\hline $\begin{array}{l}\text { Paterson. } \\
1998 \text { [29] }\end{array}$ & Australia & $\begin{array}{l}\text { Prospective } \\
\text { cohort }\end{array}$ & $\begin{array}{l}\text { Hospital- } \\
\text { based }\end{array}$ & $\begin{array}{l}\text { Laboratory } \\
\text { values }\end{array}$ & $\begin{array}{l}\text { age, duration of therapy, } \\
\text { allopurinol use, }\end{array}$ & 88 & Aminoglycoside & AKI & Low \\
\hline $\begin{array}{l}\text { Raveh, } \\
2002 \text { [30] }\end{array}$ & Israel & $\begin{array}{l}\text { Prospective } \\
\text { cohort }\end{array}$ & $\begin{array}{l}\text { Hospital- } \\
\text { based }\end{array}$ & $\begin{array}{l}\text { Laboratory } \\
\text { values }\end{array}$ & $\begin{array}{l}\text { age, gender, BMI, } \\
\text { infectious diagnosis, } \\
\text { diabetes mellitus, etc. }\end{array}$ & 209 & Aminoglycoside & AKI & Low \\
\hline $\begin{array}{l}\text { Sia, } 2018 \\
{[35]}\end{array}$ & Australia & $\begin{array}{l}\text { Retrospective } \\
\text { cohort }\end{array}$ & $\begin{array}{l}\text { Hospital } \\
\text { based }\end{array}$ & KDIGO & $\begin{array}{l}\text { Age, gender, treatment } \\
\text { indicator, ICU admission }\end{array}$ & 242 & Aminoglycoside & AKI & Low \\
\hline
\end{tabular}

KEY: AKI Acute kidney injury, DILI Drug induced liver injury, KDIGO Kidney Disease: Improving Global Outcome, AKIN Acute Kidney Injury Network, ICD-10 International Classification of Diseases version 10, CKD-EPI Chronic kidney Disease - Epidemiology collaborator, NKF National Kidney Foundation, CVD Cardiovarscular disease, ACEI Angiotensin converting enzyme Inhibitor, ARB Angiotensin II receptor blocker, NSAID Nonsteroidal anti-inflammatory drug, ICU Intensive care unit, BMI Body-Mass index

NOS risk of bias grades: low, moderate, high

34, 36] and the rest were all retrospective cohort studies. Most of the studies were hospital-based except four that were based on primary care data $[11,13,16,37]$ and three that were based on population data $[18,24,36]$. Six of the studies were carried out in the United Kingdom [11, 13, 15, 16, 37, 38], five from the United States of America [12, 14, 19, 20, 36], four from China [21, 23, $28,39]$, three from Japan [22, 25, 32], two each from Australia [29, 35], Canada [18, 24] and India [31, 34], and one each from France [17], Iran [33], Israel [30],
Singapore [27], and South Korea [26]. Out of the twenty-nine studies included for qualitative analysis, one reported on tendon rupture [36], three reported on drug-induced liver injury (DILI) [13, 26, 33], and the rest reported on acute kidney injury (AKI). After considering the classes of antimicrobials used, nine, eight and four studies reported on AKI due to aminoglycosides, glycopeptides, and macrolides, respectively. Cephalosporins, penicillin, quinolone, and trimethoprim had a couple of studies, each reporting on AKI, while nitrofurantoin had 
a single study reporting on AKI. Three studies were on antituberculosis antimicrobials, and they reported on DILI. One study on quinolones reported on tendon rupture. Table 1 below summarises the main characteristics of all the included studies.

\section{Risk of bias}

The Newcastle-Ottawa Scale (NOS) [5] with slight modifications was used to assess the risk of bias for each of the included observational studies. This tool is well validated and commonly used for assessing the risk of bias for observational studies included in a systematic review [40]. In this systematic review, the modified tool is shown in additional file 4_doc1_appendices. Table 2 above summarises the risk of bias for each included observational study. All but two of the included cohort studies had a low risk of bias. The study by Morimoto et al. [32] had moderate risk of bias due to low score on selection criteria, while the study by Giri et al. [34] had high risk of bias due to low score on comparability.

\section{Outcomes}

Out of the 29 studies included in this systematic review, two studies were not considered for meta-analysis due to their moderate or high risk of bias [32, 34]. A further two studies were not included in the meta-analysis of absolute risk because the researchers reported odds ratios only [16, 37]. However, the remaining twenty-five studies expressed the absolute risk of organ injury among exposed older adults. Eight studies [12, 15, 17, 27, 29, 30, 35, 38] reported absolute risk of AKI due to aminoglycoside exposure, with two antimicrobials reported in each of the studies by Fraisse et al. [17] and Ong et al. [27]. Eight studies reported absolute risk of AKI due to glycopeptide exposure [14, 19-21, 23, 25, 28, 39], and two studies reported the absolute risk of AKI due to macrolide exposure $[18,24]$ on two antimicrobials each. Two studies each on penicillin [11, 22], quinolones [16, 37], cephalosporins [16, 37], and trimethoprim [11, 16] also reported an absolute risk of AKI due to exposure to the respective antimicrobial. Only three studies [13, 26, 33] reported an absolute risk of DILI due to antituberculosis antimicrobials. Meta-analyses were done on absolute risks for AKI among older adults who received aminoglycoside, glycopeptide, or macrolide antimicrobials, and the respective attributable risk percentages were determined.

\section{Amino glycopeptides}

A total of 1853 participants were exposed to aminoglycosides, and 293 of them were reported to have developed antimicrobial associated AKI across the eight studies. Figure 2 below, is the funnel plot of the included studies showing a very low degree of publication bias. The random-effects model was used to meta analyse the studies to establish the overall risk of AKI among older
Table 2 Quality assessment of included studies based on the modified Newcastle-Ottawa scoring system (summarised from additional file 3, document 1)

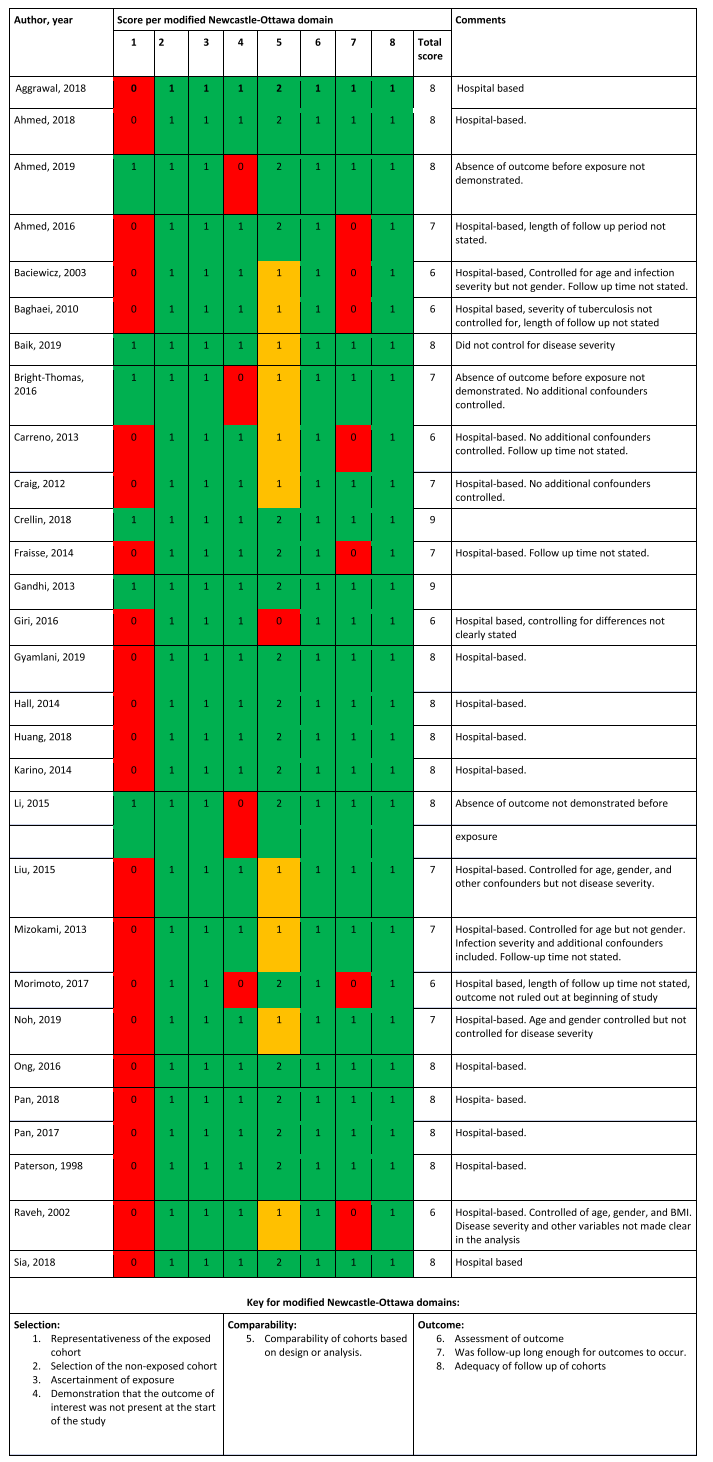

Key for modified Newcastle-Ottawa domains:

Selection:

1. Representativeness of the exposed cohort

2. Selection of the non-exposed cohort

3. Ascertainment of exposure

4. Demonstration that the outcome of interest was not present at the start of the study

Comparability:

5. Comparability of cohorts based on design or analysis.

Outcome:

6. Assessment of outcome

7. Was follow-up long enough for outcomes to occur.

8. Adequacy of follow up of cohorts

Red $=$ Failed to satisify domain (Reduces quality)

Amber $=$ Moderately satisfied domain (moderately affects quality)

Green $=$ Satisfied domain (Increases quality)

adults using aminoglycoside antimicrobials. Figure 3 below, is the forest plot of the meta-analysis and the studies show a high degree of homogeneity $\left(\mathrm{I}^{2}=34.8 \%, p=\right.$ 


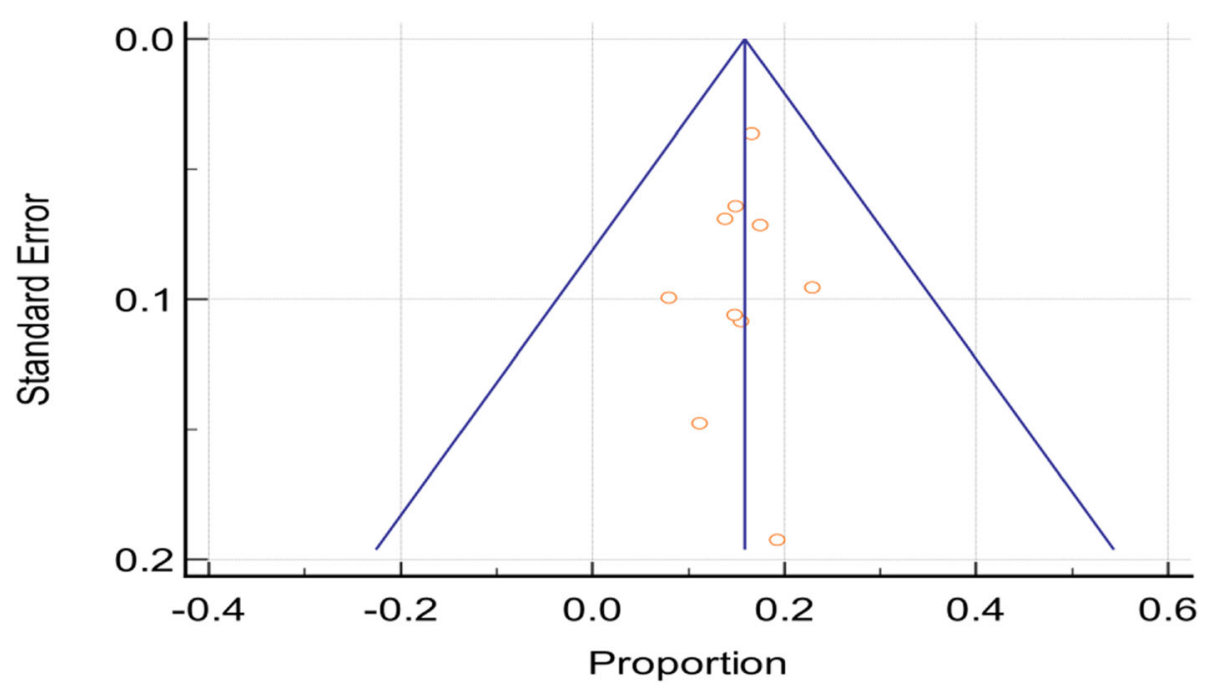

Fig. 2 Funnel plot for studies included in the meta-analysis of the proportion of acute kidney injury among older adults prescribed aminoglycosides

0.129). The overall absolute risk of AKI among older adults exposed to aminoglycosides was $15.1 \%$ (95\% CI: $12.8-17.3 \%)$. This was significantly higher $(p<0.0001)$ than the average risk of AKI among adults of 18 years and above, following aminoglycoside antimicrobial exposure (10.5\%; 95\% CI: $10.1-10.8 \%$ ) [41]. Therefore, the attributable risk per cent of AKI among older adults exposed to aminoglycosides was 30.5\% (95\% CI: 6.6-54.4\%).

\section{Glycopeptides}

A total of 23,431 participants were included in the eight studies that reported AKI due to glycopeptide exposure among older adults. Figure 4 (additional file 5, document
1) is a funnel plot showing the distribution of the studies when the standard error was plotted against the proportion of cases, and Fig. 5 (additional file 5, document 1) shows the forest plot before removing outliers, with an absolute risk of $21.4 \%$ (95\% CI: 17.1-25.7\%). After performing the leave-one-out meta-analysis, the overall absolute risk reduced significantly $(p=0.017)$ after excluding the study by Carreno et al. [14]. As shown in Fig. 6 (additional file 5, document 1), excluding other studies did not significantly impact the overall absolute risk of AKI. Figure 7 (additional file 5, document 1) shows the funnel plot following the outlier's exclusion, and Fig. 4 shows the forest plot for a random-effects

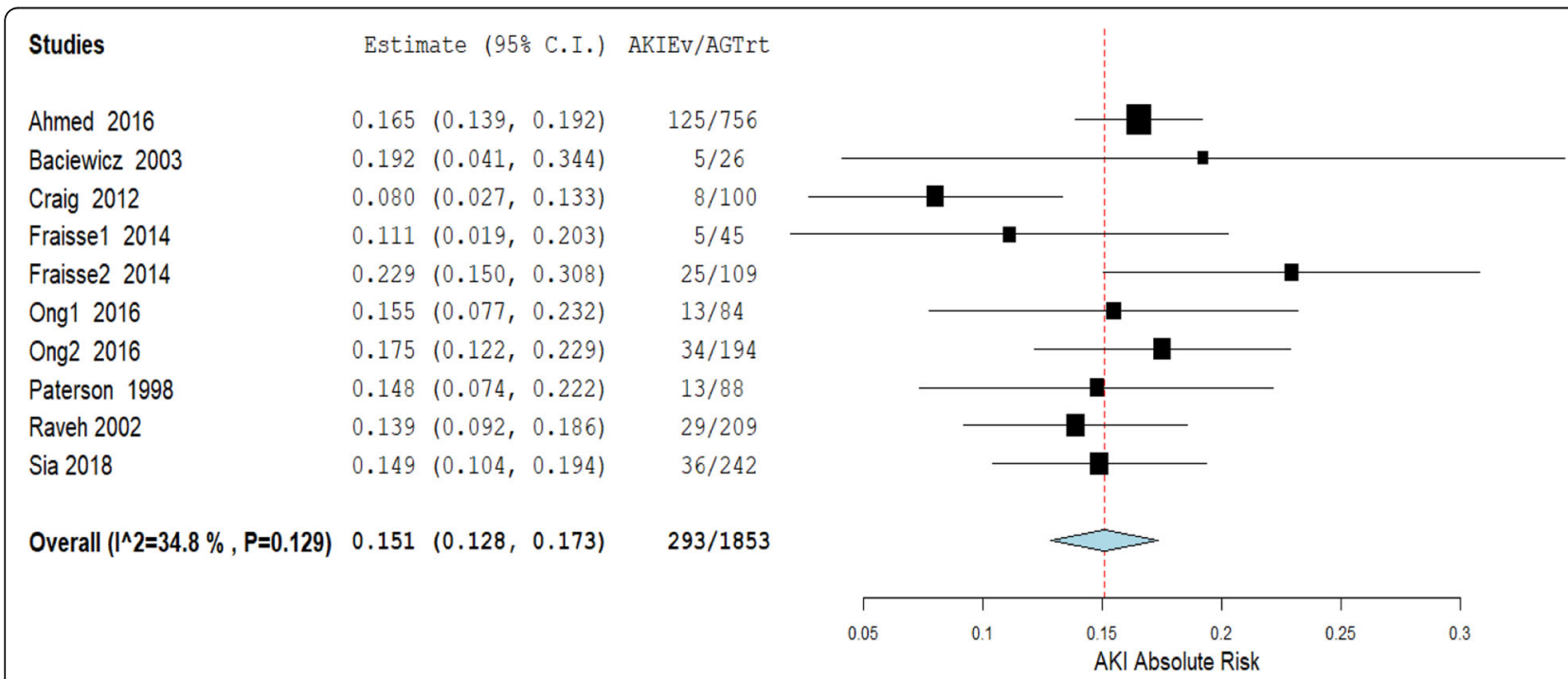

Fig. 3 Meta-analysis of the proportion of acute kidney injury among older adults prescribed aminoglycosides. AKIEv = acute kidney injury cases. AGTrt = number exposed to aminoglycosides 


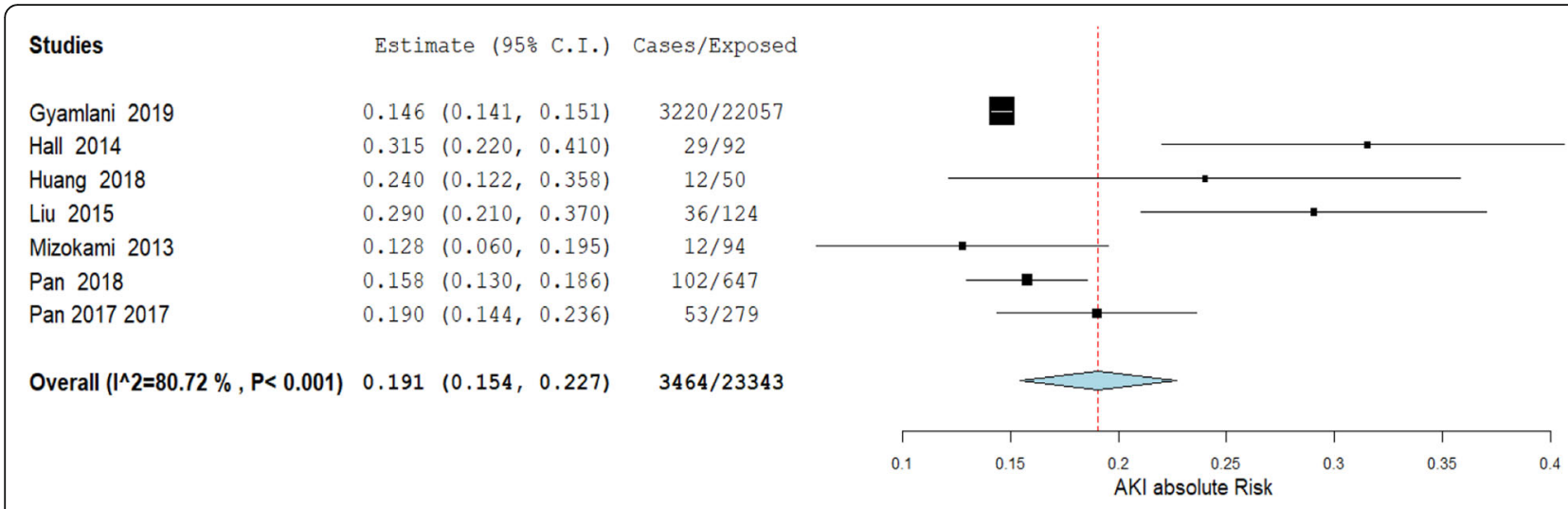

Fig. 4 Meta-analysis of the proportion of acute kidney injury among older adults prescribed glycopeptides after removing the outlier

model for the studies after removing the outlier. In this systematic review, the overall absolute risk of AKI following the use of glycopeptides is, therefore, 19.1\% (95\% CI: $15.4-22.7 \%)$. There is no significant difference ( $p=$ 0.117 ) with the established risk of AKI among adults (18 years and above) on glycopeptide antimicrobial treatment (absolute risk $=18.7 \%$; 95\% CI: 15.6-21.7\%) [42] .

\section{Macrolides}

Only two studies reported on the risk of macrolideassociated AKI, but among them, a total of 294,350 participants were involved $[18,24]$. Each of these two studies reported on two antimicrobials. The fixed-effects model was used to analyse these studies. Figure 5 is the forest plot summarising the combined outcome of these four studies. The overall risk of AKI among older adults exposed to macrolides was $0.3 \%$ (95\% CI: $0.3-0.3 \%$ ).

\section{Drug-induced liver injury (DILI)}

Only three studies, with 572 participants, reported antimicrobial-associated DILI [13, 26, 33]. In their study, Noh et al. [26] followed up 77 outpatients who were diagnosed and being treated for latent tuberculosis infection (LTBI), of which $14.3 \%$ (=11) had raised aminotransferases (the laboratory marker used to determine DILI) due to antituberculosis antimicrobials. All the participants were 65 years or above. Similarly, Bright-Thomas et al. [13] followed up 2070 adults (18 years and above) with active tuberculosis over 30 years (1981 to 2010). A subgroup of two hundred of these patients was 70 years old or more, hence included in this systematic review. Five per cent $(n=10)$ of these patients had raised aminotransferases, an indication of antimicrobial associated DILI. Baghaei et al. [33] carried out a prospective cohort study on risk of drug induced hepatitis among adult patients on antituberculosis treatment. A subgroup of participants aged 65 years or above $(n=295)$ was considered for this systematic review, of which $16.6 \%(n=49)$ had antimicrobial associated hepatitis. All three studies agreed that antimicrobial associated DILI increased with age.

\section{Subgroup analysis}

No studies included in this systematic review investigated the relative risk or odds ratio of organ injury

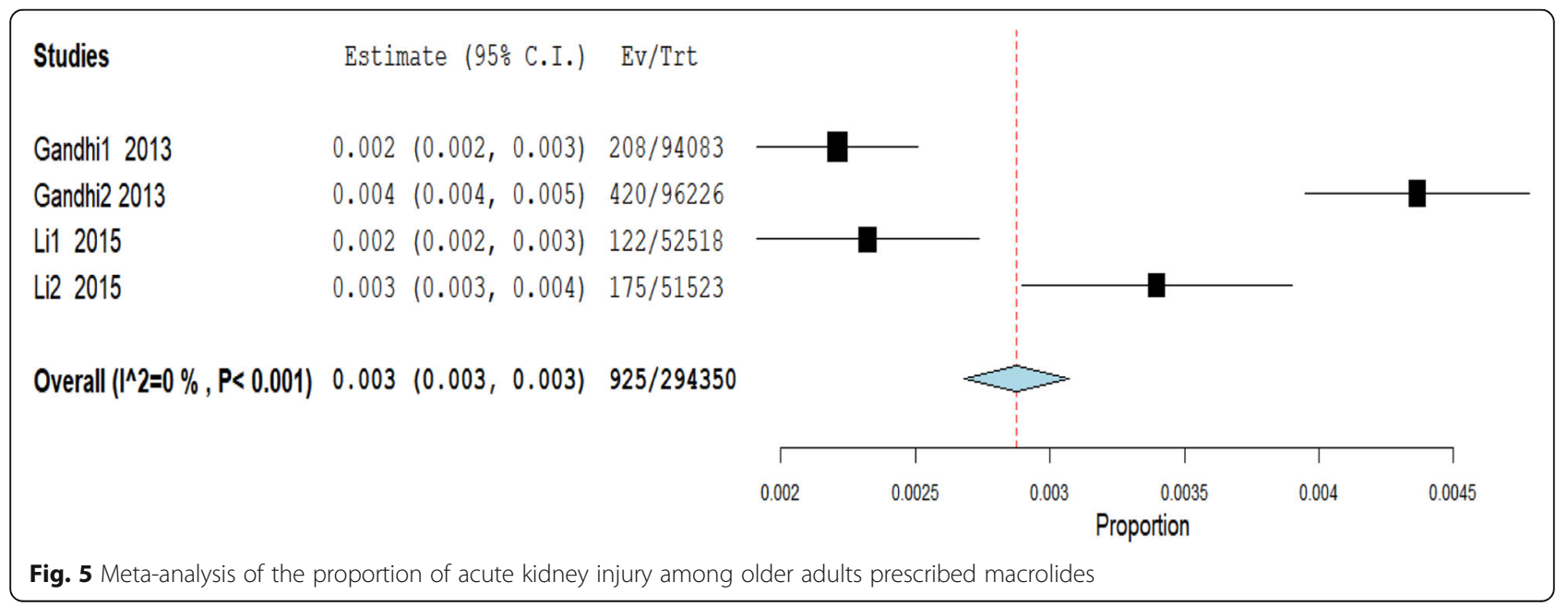


associated with broad-spectrum antimicrobials compared to narrow-spectrum antimicrobials prescribed to older adults. However, three studies in one article [37] and another separate study [11] compared the odds of AKI among older adults when empirically prescribed different antimicrobials for urinary tract infection (UTI) with empirical prescription of nitrofurantoin. In another four studies published in one research paper [16], the odds of AKI among older adults prescribed different antimicrobials following UTI were compared with the odds following amoxicillin prescription. Another five studies each had data on long term compared to short term treatment and an age group comparison of those between 65 and 79 years versus those 80 years and above.

Organ injury following empirical antimicrobial prescription for UTI Ahmed et al. [37] and Ahmed et al. [11] carried out several studies to determine the odds ratios of AKI when older adults have prescribed either cephalexin, co-amoxiclav, ciprofloxacin or trimethoprim when compared to nitrofurantoin for UTI. All exposure antimicrobials, except trimethoprim, had similar odds of causing UTI to nitrofurantoin among older adults. Trimethoprim was 1.9 times more likely to be associated with AKI when compared to nitrofurantoin (95\% CI: 1.5-2.5). Crellin et al. [16] also reported the odds ratios of AKI among the older adults when cefalexin, ciprofloxacin, nitrofurantoin and trimethoprim were compared to amoxicillin. The odds of AKI associated with nitrofurantoin or cefalexin was not significantly different to that of amoxicillin among the older adults $(\mathrm{OR}=0.89 ; 95 \% \mathrm{CI}: 0.65-1.24$, and $\mathrm{OR}=1.01$, 95\% CI: 0.74-1.37, respectively). Ciprofloxacin was 1.48 (95\% CI: 1.03-2.13) times, and trimethoprim was 1.72 (95\% CI: $1.31-2.24$ ) times more likely to be associated with AKI among older adults when compared to amoxicillin when prescribed for UTI.

Organ injury following treatment duration Among the five studies that included duration of treatment as part of their analysis, three of them $[27,29,30]$ compared up to 7 days of treatment with aminoglycosides versus more than 7 days, while the other two studies [19, 20] compared short and long term treatments with glycopeptides. In the study by Paterson et al. [29], there was a strong association of AKI among the patients who received an aminoglycoside for more than 7 days, with a risk ratio of 7.5 (95\% CI: $2.7-20.5, p=0.0001)$, when compared to those treated for seven or fewer days. Contrary, in both of their studies, Ong et al. [27] and Raveh et al. [30] found no association of risk of AKI among those who received an aminoglycoside for more than 7 days, when compared to 7 or fewer days of treatment, with relative risks of 0.99 (95\% CI: $0.58-1.68)$ and 1.35
(95\% CI: 0.68-2.68) respectively. Similarly, Gyamlani et al. [19] and Hall et al. [20] did not find any association between the risk of AKI among those who received long term glycopeptide treatment compared to short term treatment.

Organ injury according to age group Three of the five studies determined the association of AKI due to glycopeptide treatment among those at least 80 years when compared to those between 65 and 79 years [14, 20, 28]. According to Pan et al. [28], there was a significant association of AKI among those at least 80 years old and on glycopeptide therapy when compared to those between 65 and 79 years of age, with a relative risk of $2.3(95 \%$ CI: $1.5-3.8, p=0.0004)$. However, the studies by Hall et al. [20] and Carreno et al. [14] did not show any significant association of older age with AKI due to glycopeptide therapy. Similarly, both Bright-Thomas et al. [13] and Noh et al. [26] did not find any significant association of older age with DILI due to antituberculosis antimicrobials, with respective relative risks of 1.52 (95\% CI: 0.44-5.19) and 1.18 (95\% CI: 0.18-7.75).

\section{Heterogeneity and publication bias}

The included aminoglycoside studies were tested for heterogeneity and the Q-test was $11.59(p=0.2376)$, and $\mathrm{I}^{2}$ was $22.32 \%$ (95\% CI: $0.00-62.07 \%$ ). Figure 2 (above) is the funnel plot of the included studies showing a central and uniform distribution of both small and large studies around the mean, indicating a low degree of publication bias. The Egger's regression test intercept was -0.3564 (95\% CI: -2.341 to 1.628 ).

The funnel plot for studies on glycopeptide exposure (Fig. 4, additional file 5, document 1 ) was skewed to the right, with one study possibly an outlier. Figure 5 (additional file 5 , document 1 ) is the forest plot before sensitivity analysis, with an absolute risk of $21.4 \%$ (95\% CI: 17.1-25.7\%). After performing the leave-one-out metaanalysis, the overall absolute risk reduced significantly $(p=0.017)$ after excluding the study by Carreno et al. (16). As shown in Fig. 6 (additional file 5, document 1), excluding other studies did not significantly impact the overall absolute risk of AKI. The Q - test was 39.08 $(p<0.0001)$ and the $\mathrm{I}^{2}$ test was $84.65 \%$ (95\% CI: 70.1592.10\%). The funnel plot (Fig. 7, additional file 5, document 1) was skewed, and Egger's regression test intercept was 2.2664 $(p=0.0340)$.

\section{Discussion}

This is the first systematic review with meta-analysis to investigate kidney, liver, and tissue injury associated with antimicrobial exposure among older adults 65 years and above. This systematic review's primary outcome of interest was to determine the overall risk of organ injury 
among older adults who received antimicrobial therapy. Most of the studies included in our systematic review expressed the risk of kidney injury following exposure to aminoglycoside or glycopeptide antimicrobials.

\section{Antimicrobial exposure and risk of organ injury}

In our systematic review, older adults had a 15.1\% (95\% CI: 12.8-17.3\%) risk of developing AKI following exposure to aminoglycosides. Several reviews and original research studies have found a wide variation of AKI risk among patients prescribed these antimicrobials. Selby et al. [43] found a $24.4 \%$ risk of AKI among hospitalised patients associated with increased mortality. Oliveira et al. [44] found the prevalence of AKI among intensive care unit patients prescribed aminoglycosides to be as high as $58 \%$ and associated with increased mortality. On the lower end, Paquette et al. [45] found the risk of AKI from aminoglycoside exposure to be $12 \%$ after excluding children, seriously ill patients, and patients treated for less than 5 days. In a large study of 8270 participants, Fuhrman et al. [46] found the incidence of AKI associated with aminoglycosides to be only $4 \%$. Oliveira et al. [44] argued that this huge variation in prevalence is due to varying study population characteristics. However, the majority of published literature estimates the prevalence to be 10 to $20 \%$. In their systematic review and metaanalysis, Hayward et al. [41] found a $10.5 \%$ incidence of AKI among adults of 18 years to 95 years of age, from a total of 24,107 non-intensive care patients. Using this systematic review as our reference, we concluded that older adults are at significantly higher risk of AKI when prescribed aminoglycosides $(p<0.0001)$, with attributable risk from 6.6 to $54.4 \%$.

In our systematic review, glycopeptides were associated with a $19.1 \%(95 \% \mathrm{CI}: 15.4-22.7 \%)$ risk of AKI among older adults. Gyamlani et al. [19] noted that AKI incidence due to glycopeptide exposure varies between 5 and $43 \%$, depending on population and baseline risk factors. In their study, Gyamlani et al. found a $10.4 \%$ incidence of AKI among patients prescribed glycopeptides. In another study, Fuhrman et al. [46] found out that $17 \%$ of adults prescribed glycopeptides developed AKI during or post-exposure. In their systematic review and meta-analysis, Sinha Ray et al. [42] found the incidence of AKI among adults 18 years and above to be $18.7 \%$ (95\% CI: 15.6-21.7\%). Using this study as our baseline, there was no significant difference with older adults' findings $(p=0.117)$. We, therefore, concluded that older adults of 65 years and above have a similar risk of developing AKI when prescribed glycopeptides as the general adult population of 18 years and above.

Only $0.3 \%$ of AKI was associated with macrolides to older adults in our systematic review. This is a rare outcome; therefore, there are not many studies done to explore this relationship. Similarly, we only found three studies that reported antimicrobial associated DILI among older adults. The number of studies was too small to perform a meaningful meta-analysis. However, the three studies had an average of $12.3 \%$ risk of antimicrobial associated DILI among older adults. Although fewer studies report antimicrobial-associated DILI among older adults, several studies have been reported on the general adult population. In a Swedish study, 27\% of DILI was attributed to antimicrobials [47]. Similar studies were also done in Spain [48], India [49] and the United States of America [50] and found 32, 65, and $45 \%$ of DILI associated with antimicrobials, respectively. Several antimicrobials have been implicated, but most commonly were amoxicillin-clavulanic acid [48, 50], erythromycin [51] and nitrofurantoin [50].

\section{Antimicrobial exposure and risk of organ injury by the duration}

In our systematic review, the three studies that reported on the association of AKI with aminoglycoside treatment duration did not agree on their findings. Paterson et al. [29] found a relative risk ratio of 7.5 when patients were prescribed aminoglycosides for more than 7 days compared to those less than 7 days. However, Raveh et al. [30] reported an increased risk of AKI when aminoglycoside treatment was prolonged to 11 days and above. However, Selby et al. [43] did not find any association between AKI and treatment duration.

\section{Antimicrobial exposure and risk of organ injury by age}

We did not find any studies that assessed the association of aminoglycoside prescription with AKI by age group. However, some authors have found an increased risk of AKI with age from the general adult population [43]. Only one out of the three studies in our systematic review showed a significant association of age with AKI following glycopeptide prescription to older adults [28]. In their systematic review, van $\mathrm{Hal}$ et al. [52] found out that AKI risk following glycopeptide prescription increases with age if patients are exposed for more than 7 days.

\section{Heterogeneity and publication bias}

In our systematic review, we used the random-effects model to summarise the risk of AKI among older adults prescribed aminoglycosides and glycopeptides. Several authors, for example, Ioannidis [53] and Huedo-Medina et al. [54], decide on whether to use a fixed-effect or random-effects model based on heterogeneity results. However, we chose our model before heterogeneity testing, based on recommendations by Borenstein et al. [55] and further supported by Spinelli and Pandis [56]. Our studies were drawn from across the globe and were 
carried out under different conditions, so we assumed they were not identical. In this instance, heterogeneity testing helped us determine whether these studies have enough in common to be meta-analysed [56]. We also used the funnel plot [7] and Egger's regression test [8] to assess our studies' publication bias. We meta-analysed nine studies on aminoglycosides and seven studies on glycopeptides.

\section{Strengths of our study}

The study focused on older adults aged 65 and above, who are usually underrepresented in randomised controlled trials. Real-world data, which is more representative of the older adults prescribed antimicrobials, was used. The quality of each included study was thoroughly assessed on representativeness on study participants, ascertainment of exposure of interest, absence of outcome of interest at the beginning of the study, control of possible confounders including age, gender, and severity of the disease, outcome assessment, and adequacy of follow up for outcome to occur.

Most of the included studies shared some common strengths. All the studies considered a wide range of possible confounders and adjusted for them accordingly in their analyses. Well known and validated tools were used to ascertain the presence or absence of outcomes. For example, the international classification of diseases version 10 (ICD-10) was used by authors whose study settings were primary care or population-based [11, 16, 18, 24, 37]. Most hospital-based studies used tools that relied on laboratory markers for organ injury, such as a two-fold rise in serum creatinine or doubling of liver transaminases. Some of the tools used include the acute kidney injury network (AKIN) [14, 21, 38] and kidney disease, improving global outcome (KDIGO) [27, 28, 39]. Only three studies $[13,24,37]$ did not describe how they determined the absence of outcome of interest at the beginning of their studies.

\section{Limitations of our study}

Despite their strengths, the included studies in this systematic review also came with their weaknesses. All the included studies, except five [13, 16, 18, 24, 37], were hospital-based. The general major drawback of using hospitalised patients is the generalisability of the findings [57]. Most of our data were obtained from subgroups embedded in larger studies. This led to our data lacking detailed information, for example, gender differences among the older adults, since we relied heavily on limited results from tables or otherwise.

\section{Conclusion}

In conclusion, the findings from this systematic review and meta-analysis suggest that there is a significantly increased risk of AKI among older adults when compared to the general adult population following exposure to aminoglycosides. Our results indicated that the risk of AKI in older adults is $15.1 \%$ (95\% CI: $12.8-17.3$ ). The risk of AKI following exposure to glycopeptides for older adults is similar to that previously found in the general adult population. We did not get enough studies to determine whether age or duration of treatment influences the risk of AKI among older adults.

\section{Abbreviations}

ACEl: Angiotensin converting enzyme Inhibitor; AKI: Acute kidney injury; AKIN: Acute Kidney Injury Network; ARB: Angiotensin II receptor blocker; CKD-EPI: Chronic kidney Disease - Epidemiology collaborator; DILI: Druginduced liver injury; DILI: Drug induced liver injury; ICD-10: International Classification of Diseases version 10; ICU: Intensive care unit; BMI- Body-Mass index; KDIGO: Kidney Disease: Improving Global Outcome; LTBI: Latent tuberculosis infection; NKF: National Kidney Foundation; CVD: Cardiovarscular disease; NSAID: Nonsteroidal anti-inflammatory drug; UTI: Urinary tract infection

\section{Supplementary Information}

The online version contains supplementary material available at https://doi. org/10.1186/s12877-021-02512-3.

Additional file 1. Risk of antimicrobial-associated organ injury among older adults: A systematic Review and Meta-Analysis.

Additional file 2. Risk factors for nephrotoxicity in elderly patients receiving once-daily aminoglycosides.

Additional file 3. Study appraisals - Risk of Bias assessment using Newcastle-Ottawa scale.

Additional file 4: Appendix 1. Modified Newcastle-Ottawa Scale: Cohort Studies tool.

Additional file 5. Funnel plots and leave-one-meta-analysis figures.

\section{Acknowledgements}

The authors would like to thank Marylou Francoise, the acting librarian for the Faculty of Science at University of Bath, for her immense help in training and supporting article search strategies from various databases.

\section{Authors' contributions}

$\mathrm{TC}$ is the first author of the systematic review and meta-analysis paper. He led all the stages of the development and writing of this script. At the same time, PN and SR supervised and contributed to developing plans for searching, screening, extracting, and writing phases. All authors have read and approved the manuscript.

\section{Funding}

No funding was received for this systematic review and meta-analysis..

\section{Availability of data and materials}

All data generated or analysed during this study are included in this published article (and its supplementary information files).

\section{Declarations}

Ethics approval and consent to participate Not applicable.

Consent for publication

Not applicable.

Competing interests

The authors declare that they have no competing interests. 


\section{Author details}

'Department of Pharmacy and Pharmacology \& Centre for Therapeutic Innovation, University of Bath, Bath BA2 7AY, UK. ${ }^{2}$ Department of Mathematical Science, University of Bath, Bath BA2 7AY, UK.

\section{Received: 18 March 2021 Accepted: 10 September 2021} Published online: 01 November 2021

\section{References}

1. Augustine $\mathrm{S}$, Bonomo RA. Taking stock of infections and antibiotic resistance in the elderly and long-term care facilities: a survey of existing and upcoming challenges. Eur J Microbiol Immunol (Bp). 2011;1(3):190-7. https://doi.org/10.1556/EuJMI.1.2011.3.2.

2. Esme M, Topeli A, Yavuz BB, Akova M. Infections in the Elderly Critically-III Patients. Front Med (Lausanne). 2019;6:118.

3. Giarratano A, Green SE, Nicolau DP. Review of antimicrobial use and considerations in the elderly population. Clin Interv Aging. 2018;13:657-67. https://doi.org/10.2147/CIA.S133640

4. Martin K, Bégaud B, Latry P, Miremont-Salamé G, Fourrier A, Moore N. Differences between clinical trials and postmarketing use. Br J Clin Pharmacol. 2004:57(1):86-92. https://doi.org/10.1046/j.1365-2125.2003.01953.x.

5. Wells GA SB, O'Connell D, Peterson J, Welch V, Losos M, Tugwell P. The Newcastle-Ottawa Scale (NOS) for assessing the quality of nonrandomised studies in meta-analyses [cited 202001 October]. Available from: http:// www.ohri.ca/programs/clinical_epidemiology/oxford.asp.

6. Higgins JP, Thompson SG, Deeks JJ, Altman DG. Measuring inconsistency in meta-analyses. BMJ. 2003;327(7414):557-60. https://doi.org/10.1136/bmj.327. 7414.557.

7. Sterne JA, Egger M. Funnel plots for detecting bias in meta-analysis: guidelines on choice of axis. J Clin Epidemiol. 2001;54(10):1046-55. https:// doi.org/10.1016/S0895-4356(01)00377-8.

8. Egger M, Davey Smith G, Schneider M, Minder C. Bias in meta-analysis detected by a simple, graphical test. BMJ. 1997;315(7109):629-34. https:// doi.org/10.1136/bmj.315.7109.629

9. DerSimonian R, Laird N. Meta-analysis in clinical trials. Control Clin Trials. 1986;7(3):177-88. https://doi.org/10.1016/0197-2456(86)90046-2.

10. Wallace BC, Dahabreh IJ, Trikalinos TA, Lau J, Trow P, Schmid CH. Closing the gap between methodologists and end-users: $\mathrm{R}$ as a computational Back-end. J Stat Softw. 2012;49(5). https://doi.org/10.18637/jss.v049.i05.

11. Ahmed H, Farewell D, Francis NA, Paranjothy S, Butler CC. Risk of adverse outcomes following urinary tract infection in older people with renal impairment: retrospective cohort study using linked health record data. PLoS Med. 2018;15(9):e1002652. https://doi.org/10.1371/journal. pmed.1002652.

12. Baciewicz AM, Sokos DR, Cowan RI. Aminoglycoside-associated nephrotoxicity in the elderly. Ann Pharmacother. 2003:37(2):182-6. https:// doi.org/10.1177/106002800303700203.

13. Bright-Thomas RJ, Gondker AR, Morris J, Ormerod LP. Drug-related hepatitis in patients treated with standard anti-tuberculosis chemotherapy over a 30year period. Int J Tuberc Lung Dis. 2016;20(12):1621-4. https://doi.org/10. 5588/ijtld.16.0370

14. Carreno JJ, Jaworski A, Kenney RM, Davis SL. Comparative incidence of nephrotoxicity by age group among adult patients receiving Vancomycin. Infect Dis Ther. 2013;2(2):201-8. https://doi.org/10.1007/s40121-013-0022-6.

15. Craig P, Starks I, Bancroft G, Roberts P. Is prophylactic gentamicin associated with acute kidney injury in patients undergoing surgery for fractured neck of femur? Injury. 2012;43(12):2152-5. https://doi.org/10.1 016/j.injury.2012.08.005.

16. Crellin E, Mansfield KE, Leyrat C, Nitsch D, Douglas IJ, Root A, et al. Trimethoprim use for urinary tract infection and risk of adverse outcomes in older patients: cohort study. BMJ. 2018;360:k341. https://doi.org/10.1136/ bmj.k341.

17. Fraisse T, Gras Aygon C, Paccalin M, Vitrat V, De Wazieres B, Baudoux V, et al. Aminoglycosides use in patients over 75 years old. Age Ageing. 2014;43(5): 676-81. https://doi.org/10.1093/ageing/afu023.

18. Gandhi S, Fleet JL, Bailey DG, McArthur E, Wald R, Rehman F, et al. Calciumchannel blocker-clarithromycin drug interactions and acute kidney injury. JAMA. 2013;310(23):2544-53. https://doi.org/10.1001/jama.2013.282426.

19. Gyamlani G, Potukuchi PK, Thomas F, Akbilgic O, Soohoo M, Streja E, et al. Vancomycin-associated acute kidney injury in a large veteran population. Am J Nephrol. 2019;49(2):133-42. https://doi.org/10.1159/000496484.
20. Hall RG, Blaszczyk AT, Thompson KA, Brouse SD, Giuliano CA, Frei CR, et al. Impact of empiric weight-based vancomycin dosing on nephrotoxicity and mortality in geriatric patients with methicillin-resistant Staphylococcus aureus bacteraemia. J Clin Pharm Ther. 2014;39(6):653-7. https://doi.org/1 $0.1111 /$ jcpt.12203.

21. Huang M, Wu H, Zhou J, Xu M, Zhou S. Efficacy of Vancomycin on grampositive bacterial infection in elderly critical patients and risk factors associated with nephrotoxicity. Arch Iran Med. 2018;21(8):349-55.

22. Karino F, Nishimura N, Ishihara N, Moriyama H, Miura K, Hamaguchi S, et al. Nephrotoxicity induced by piperacillin-tazobactam in late elderly Japanese patients with nursing and healthcare associated pneumonia. Biol Pharm Bull. 2014;37(12):1971-6. https://doi.org/10.1248/bpb.b14-00362.

23. Liu Y, Yin Y, Liu XZ, Yao HJ, Li LX, Chen JH, et al. Retrospective analysis of vancomycin nephrotoxicity in elderly Chinese patients. Pharmacology. 2015; 95(5-6):279-84. https://doi.org/10.1159/000381783.

24. Li DQ, Kim R, McArthur E, Fleet JL, Bailey DG, Juurlink D, et al. Risk of adverse events among older adults following co-prescription of clarithromycin and statins not metabolized by cytochrome P450 3A4. CMAJ. 2015:187(3):174-80. https://doi.org/10.1503/cmaj.140950.

25. Mizokami F, Shibasaki M, Yoshizue Y, Noro T, Mizuno T, Furuta K. Pharmacodynamics of vancomycin in elderly patients aged 75 years or older with methicillin-resistant Staphylococcus aureus hospital-acquired pneumonia. Clin Interv Aging. 2013;8:1015-21. https://doi.org/10.2147/CIA. S50238.

26. Noh CS, Kim HI, Choi H, Kim Y, Kim CH, Choi JH, et al. Completion rate of latent tuberculosis infection treatment in patients aged 65 years and older. Respir Med. 2019;157:52-8. https://doi.org/10.1016/j.rmed.2019.09.004.

27. Ong LZ, Tambyah PA, Lum LH, Low ZJ, Cheng I, Murali TM, et al. Aminoglycoside-associated acute kidney injury in elderly patients with and without shock. J Antimicrob Chemother. 2016;71(11):3250-7. https://doi. org/10.1093/jac/dkw296

28. Pan K, Ma L, Xiang Q, Li X, Li H, Zhou Y, et al. Vancomycin-associated acute kidney injury: a cross-sectional study from a single center in China. PLoS One. 2017:12(4):e0175688. https://doi.org/10.1371/journal.pone.0175688.

29. Paterson $\mathrm{DL}$, Robson JM, Wagener MM. Risk factors for toxicity in elderly patients given aminoglycosides once daily. J Gen Intern Med. 1998;13(11): 735-9. https://doi.org/10.1046/j.1525-1497.1998.00224.X.

30. Raveh D, Kopyt M, Hite Y, Rudensky B, Sonnenblick M, Yinnon AM. Risk factors for nephrotoxicity in elderly patients receiving once-daily aminoglycosides. QJM. 2002;95(5):291-7. https://doi.org/10.1093/qjmed/95. 5.291.

31. Aggarwal R, Dewan A. Comparison of nephrotoxicity of Colistin with Polymyxin B administered in currently recommended doses: a prospective study. Ann Clin Microbiol Antimicrob. 2018;17(1):15. https://doi.org/10.1186/ s12941-018-0262-0.

32. Morimoto T, Nagashima H, Morimoto Y, Tokuyama S. Frequency of acute kidney injury caused by Tazobactam/Piperacillin in patients with pneumonia and chronic kidney disease: a retrospective observational study. Yakugaku Zasshi. 2017:137(9):1129-36. https://doi.org/10.1248/yakushi.17-00002.

33. Baghaei $P$, Tabarsi $P$, Chitsaz E, Saleh M, Marjani M, Shemirani S, et al. Incidence, clinical and epidemiological risk factors, and outcome of druginduced hepatitis due to antituberculous agents in new tuberculosis cases. Am J Ther. 2010;17(1):17-22. https://doi.org/10.1097/MJT.0b013e31818f9eae.

34. Giri VP, Giri OP, Bajracharya S, Khan FA, Sinha SP, Kanodia S, et al. Risk of acute kidney injury with amikacin versus gentamycin both in combination with metronidazole for surgical prophylaxis. J Clin Diagn Res. 2016;10(1): FC09-12. https://doi.org/10.7860/JCDR/2016/15621.7099.

35. Sia CS, Ananda-Rajah MR, Adler NR, Yi-Wei B, Liew D, Tong EY, et al. Renal safety of short-term empiric gentamicin therapy in aged patients. Australas J Ageing. 2018;37(3):227-31. https://doi.org/10.1111/ajag.12541.

36. Baik S, Lau J, Huser V, McDonald CJ. Association between tendon ruptures and use of fluoroquinolone, and other oral antibiotics: A 10-year retrospective study of 1 million US senior Medicare beneficiaries. BMJ Open. 2020:10(12):e034844.

37. Ahmed H, Farewell D, Francis NA, Paranjothy S, Butler CC. Choice of Empirical Antibiotic Therapy and Adverse Outcomes in Older Adults With Suspected Urinary Tract Infection: Cohort Study. Open Forum Infect Dis. 2019:6(3):ofz039.

38. Ahmed I, Khan MA, Allgar V, Mohsen A. The effectiveness and safety of two prophylactic antibiotic regimes in hip-fracture surgery. Eur J Orthop Surg Traumatol. 2016;26(5):483-92. https://doi.org/10.1007/s00590-016-1794-7. 
39. Pan KM, Wu Y, Chen C, Chen ZZ, Xu JA, Cao L, et al. Vancomycin-induced acute kidney injury in elderly Chinese patients: a single-Centre crosssectional study. Br J Clin Pharmacol. 2018;84(8):1706-18. https://doi.org/1 $0.1111 /$ bcp.13594.

40. Moskalewicz A, Oremus M. No clear choice between Newcastle-Ottawa scale and appraisal tool for cross-sectional studies to assess methodological quality in cross-sectional studies of health-related quality of life and breast cancer. J Clin Epidemiol. 2020;120:94-103. https://doi.org/10.1016/j.jclinepi.2 019.12.013.

41. Hayward RS, Harding J, Molloy R, Land L, Longcroft-Neal K, Moore D, et al. Adverse effects of a single dose of gentamicin in adults: a systematic review. Br J Clin Pharmacol. 2018;84(2):223-38. https://doi.org/10.1111/ bcp.13439.

42. Sinha Ray A, Haikal A, Hammoud KA, Yu AS. Vancomycin and the risk of AKI: a systematic review and Meta-analysis. Clin J Am Soc Nephrol. 2016;11(12): 2132-40. https://doi.org/10.2215/CJN.05920616.

43. Selby NM, Shaw S, Woodier N, Fluck RJ, Kolhe NV. Gentamicin-associated acute kidney injury. Qjm. 2009;102(12):873-80. https://doi.org/10.1093/ gjmed/hcp143.

44. Oliveira JF, Silva CA, Barbieri CD, Oliveira GM, Zanetta DM, Burdmann EA. Prevalence and risk factors for aminoglycoside nephrotoxicity in intensive care units. Antimicrob Agents Chemother. 2009;53(7):2887-91. https://doi. org/10.1128/AAC.01430-08.

45. Paquette $F$, Bernier-Jean A, Brunette V, Ammann $H$, Lavergne V, Pichette V, et al. Acute kidney injury and renal recovery with the use of aminoglycosides: a large retrospective study. Nephron. 2015;131(3):153-60 https://doi.org/10.1159/000440867.

46. Fuhrman DY, Kane-Gill S, Goldstein SL, Priyanka P, Kellum JA. Acute kidney injury epidemiology, risk factors, and outcomes in critically ill patients 16-25 years of age treated in an adult intensive care unit. Ann Intensive Care. 2018;8(1):26. https://doi.org/10.1186/s13613-018-0373-y.

47. Björnsson E, Olsson R. Outcome and prognostic markers in severe druginduced liver disease. Hepatology. 2005;42(2):481-9. https://doi.org/10.1002/ hep.20800.

48. Andrade RJ, Lucena Ml, Fernández MC, Pelaez G, Pachkoria K, García-Ruiz E, et al. Drug-induced liver injury: an analysis of 461 incidences submitted to the Spanish registry over a 10-year period. Gastroenterology. 2005;129(2): 512-21. https://doi.org/10.1016/j.gastro.2005.05.006.

49. Devarbhavi H, Dierkhising R, Kremers WK, Sandeep MS, Karanth D, Adarsh CK. Single-center experience with drug-induced liver injury from India: causes, outcome, prognosis, and predictors of mortality. Am J Gastroenterol. 2010;105(11):2396-404. https://doi.org/10.1038/ajg.2010.287.

50. Chalasani N, Bonkovsky HL, Fontana R, Lee W, Stolz A, Talwalkar J, et al. Features and Outcomes of 899 Patients With Drug-Induced Liver Injury: The DILIN Prospective Study. Gastroenterology. 2015;148(7):1340-52.e7.

51. Björnsson ES, Hoofnagle JH. Categorization of drugs implicated in causing liver injury: critical assessment based on published case reports. Hepatology 2016;63(2):590-603. https://doi.org/10.1002/hep.28323.

52. van Hal SJ, Paterson DL, Lodise TP. Systematic review and meta-analysis of vancomycin-induced nephrotoxicity associated with dosing schedules that maintain troughs between 15 and 20 milligrams per liter. Antimicrob Agents Chemother. 2013;57(2):734-44. https://doi.org/10.1128/AAC.01 568-12.

53. Ioannidis JP. Interpretation of tests of heterogeneity and bias in metaanalysis. J Eval Clin Pract. 2008;14(5):951-7. https://doi.org/10.1111/j.1365-2 753.2008.00986.x.

54. Huedo-Medina TB, Sánchez-Meca J, Marín-Martínez F, Botella J. Assessing heterogeneity in meta-analysis: Q statistic or 12 index? Psychol Methods. 2006;11(2):193-206, https://doi.org/10.1037/1082-989X.11.2.193.

55. Borenstein M, Hedges LV, Higgins JP, Rothstein HR. A basic introduction to fixed-effect and random-effects models for meta-analysis. Res Synth Methods. 2010;1(2):97-111. https://doi.org/10.1002/jrsm.12.

56. Spineli LM, Pandis N. Meta-analysis: random-effects model. Am J Orthod Dentofac Orthop. 2020;157(2):280-2. https://doi.org/10.1016/.jajodo.2019.10.007.

57. Kukull WA, Ganguli M. Generalizability: the trees, the forest, and the lowhanging fruit. Neurology. 2012;78(23):1886-91. https://doi.org/10.1212/WNL. 0b013e318258f812.

\section{Publisher's Note}

Springer Nature remains neutral with regard to jurisdictional claims in published maps and institutional affiliations.

Ready to submit your research? Choose BMC and benefit from:

- fast, convenient online submission

- thorough peer review by experienced researchers in your field

- rapid publication on acceptance

- support for research data, including large and complex data types

- gold Open Access which fosters wider collaboration and increased citations

- maximum visibility for your research: over $100 \mathrm{M}$ website views per year

At BMC, research is always in progress.

Learn more biomedcentral.com/submissions 